\title{
Avena eriantha Durieu (Gramineae) en la cuenca del Duero (España)
}

\author{
Jesús Antonio Lázaro-Bello
}

\author{
Correspondencia: \\ C/ Madre de Dios № 15, 1 D. \\ 47011-Valladolid (España). \\ E-mail: chuchijalb@hotmail.com \\ Recibido: 20 diciembre 2019 \\ Aceptado: 11 febrero 2020 \\ Publicado on-line: 13 mayo 2020 \\ Editado por: Antonio Galán de \\ Mera
}

\begin{abstract}
Resumen
Se informa del hallazgo de Avena eriantha Durieu (Gramineae) en la provincia de Valladolid (Cuenca del Duero, España). Además, se aportan datos y fotografías de la especie.
\end{abstract}

Palabras clave. Corología. Flora Vascular. Poaceae. España. Valladolid.

\begin{abstract}
Avena eriantha Durieu (Gramineae) in the Duero Basin (Spain)

The presence of Avena eriantha Durieu (Gramineae) in Valladolid province (Duero Basin, Spain) is reported. Besides data and photographs of the species are provided.
\end{abstract}

Key words. Chorology. Vascular Flora. Poaceae. Spain. Valladolid.

\begin{abstract}
Avena eriantha Durieu, Rev. Bot. Recueil Mens. 1: 360 (1845)

VALLADOLID. Olmos de Esgueva, 30TUM7614, 860 m.s.n.m., claros en mancha mixta de encina y quejigo en paramera calcárea, 08-VI-2018, J.A. Lázaro-Bello (MA-01-00940105).
\end{abstract}

Avena eriantha Durieu (IPNI, 2019; POWO, 2019) es una gramínea con biotipo de terófito y distribución mediterránea e irano-turaniana: España, norte de África, Europa desde Grecia hasta la península de Crimea, y la zona centro-oeste asiática hasta Afganistán y Kirguistán (Baum, 1977; Dogan, 1985; Romero-Zarco, 1996, 2011). En la península Ibérica, por ahora, la especie ha sido localizada en las provincias de Almería, Cuenca, Guadalajara, Madrid, Málaga, Murcia, Toledo y Zaragoza (Carrasco et al., 1986; Cebolla-Lozano \& PostigoMijarra, 1996; Hernández-Palacios, 2013; Montserrat-Recorder \& Hernández-Cardona, 1983; Paunero, 1958; Pyke, 2005; Sánchez-Gómez et al., 2018), pareciendo que la dada para Bilbao (Pyke, 2005) es una cita dudosa o errónea. A este respecto, Romero-Zarco (1983, 1990, 1996, 2015), en todos aquellos de sus trabajos que ha mencionado la especie, siempre ha ido haciendo un recopilatorio de la situación geográfica conocida en la península hasta dicho momento.

Aunque la agrostóloga Elena Paunero (Paunero, 1958) fue la primera que señaló la especie en España (sub Avena pilosa M.Bieb.), a partir de la revisión de pliegos recolectados a principios del siglo $\mathrm{XX}$, la especie ha pasado bastante desapercibida por su confusión con otras. De hecho, allí en donde se han confeccionado catálogos florísticos provinciales, bien de toda la flora vascular, bien solamente de la familia de las gramíneas, y con el taxón ya conocido en la provincia en cuestión, ha sido señalado en algunos casos (Cabezudo et al., 1990; Cebolla-Lozano et al., 1997; Morales, 2003; Prieto-Bocanegra \& Fernández-López, 1992,), pero no en otros (Carrasco et al., 1997; GonzálezBernáldez, 1997; Sanz-Elorza, 2006). Ni siquiera en su día, en Flora Europaea (Tutin et al., 1980), el taxón fue indicado en la península Ibérica.

A partir de los ejemplares recolectados, de los que hay muestras depositadas en el herbario del Real Jardín Botánico de Madrid (MA), vamos a apuntar algunas características observadas, que sirven para complementar las dadas a partir de los pocos ejemplares recogidos a nivel nacional (ANTHOS, 2019; Paunero, 1958; Romero-Zarco, 1996, 2011), o las mencionadas en parte de la literatura internacional más representativa (Baum, 1977; Dogan, 1985; POWO, 2019; Rocha-Alfonso, 1980). Nuestros ejemplares son plantas anuales, con tallos hasta de $75 \mathrm{~cm}$, solitarios o fasciculados, estriados y glabros, salvo en las zonas de los nudos en donde pueden aparecer algunos pelos cortos. Las hojas y las vainas basales son muy pelosas, con pelos patentes o algo retrorsos, y se van haciendo glabrescentes al ir ascendiendo por el tallo. La pilosidad de las hojas es más abundante en los bordes que en las caras. El limbo de las hojas basales mide $3(5) \mathrm{mm}$ de anchura y hasta unos 8 $\mathrm{cm}$ de longitud, siendo las hojas medias y superiores mucho más pequeñas. La lígula, de unos $2 \mathrm{~mm}$, es algo obtusa. La panícula, hasta de $15 \mathrm{~cm}$ de longitud y raquis lampiño, presenta en los individuos fasciculados ramas inferiores largas, hasta en grupos de cuatro por nudo (haciéndose menos congestas hacia la parte superior), con pedicelos hasta de $5,5 \mathrm{~cm}$ y pilosidad antrorsa. En los individuos unicaules las ramas son mucho más cortas, hasta de $3 \mathrm{~cm}$ de longitud, y son solitarias o geminadas en cada nudo. Espiguillas hasta de 25 
$\mathrm{mm}$ de longitud, con 2-3 flores (y, a veces, apuntando un esbozo de una cuarta flor). La raquilla se desarticula en la madurez por encima de las glumas, pero no entre las flores. Glumas marcadamente desiguales, con una gluma inferior que es 1/2 (2/3) de la superior (Fig.1A). Ambas glumas son glabras, la superior con 7 nervios y hasta de unos $23 \mathrm{~mm}$, la inferior normalmente con 5 nervios y hasta unos $13 \mathrm{~mm}$ de longitud. El lema de la flor inferior, que es el más grande, mide hasta unos $20 \mathrm{~mm}$ (incluyendo las súbulas). En la mitad inferior es glabro y en la mitad superior va pasando progresivamente de escábrido a peloso (Fig. 1B). Las súbulas 0 arístulas (que presentan pilosidad antrorsa) de la flor inferior son hasta de unos $7 \mathrm{~mm}$, mucho más largas que las de la segunda flor, que solo alcanzan unos $3 \mathrm{~mm}$ de longitud. Sólo las dos flores inferiores están subuladas y también son las únicas con lemas aristados. Aristas en posición medio-dorsal, geniculadas, hasta de $45 \mathrm{~mm}$ de longitud, retorcidas en su mitad inferior, densamente pelosas hasta el codo, y de cortamente pelosas a escábridas en la mitad superior. Pálea bífida, alcanzando 3/4 de la longitud del lema, biaquillada, con quillas aladas y densamente ciliadas. Cariópside pelosa. Callo linear, de algo menos de $3 \mathrm{~mm}$ de longitud y densamente peloso. Los pelos del callo hasta de $5 \mathrm{~mm}$, los de la base de la segunda flor más largos, hasta de $8 \mathrm{~mm}$. Artejo de la raquilla entre las dos flores inferiores lampiña.

Partiendo del hecho de que en las diversas claves de determinación que tratan el género Avena L. siempre se utiliza la notoria diferencia entre el tamaño de las glumas, para distinguir la especie que nos ocupa de otras, y tomando como referencia la descripción de Paunero (1958), otros caracteres destacables de los ejemplares aquí aportados son, por una parte, la marcada diferencia en la longitud de las súbulas o arístulas de las dos flores inferiores (Fig. 1C), y, por otra parte, la también distinta longitud de los pelos basales de las dos primeras flores (Fig. 1D).

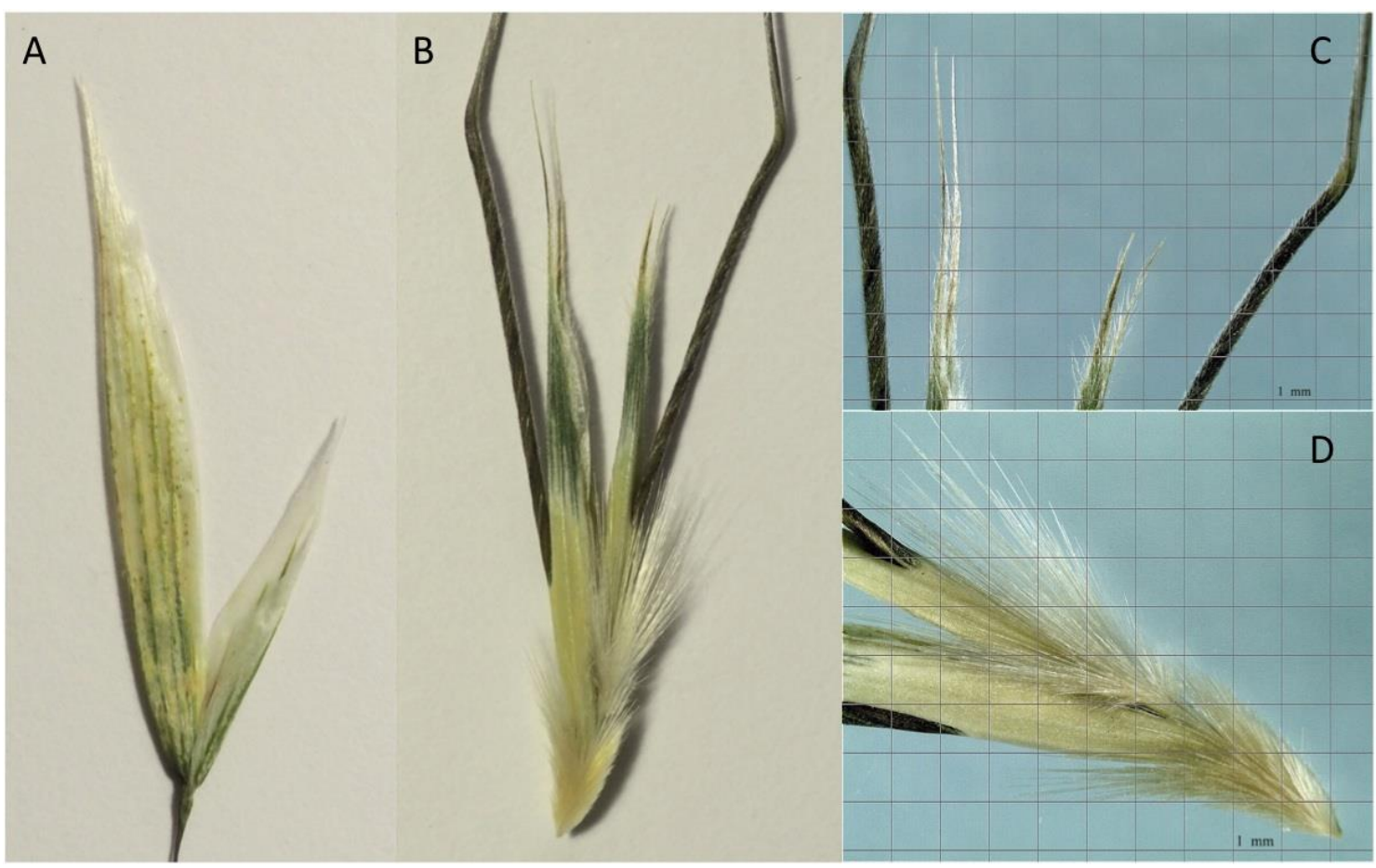

Figura 1. Caracteres de la espiguilla de Avena eriantha. A: Gluma superior (a la izquierda), de unos 23 mm de longitud, y gluma inferior (a la derecha), B: Espiguilla (de unos $25 \mathrm{~mm}$ de longitud), sin las glumas, C: Lemas aristulados, D: Callo peloso.

Figure 1. Characters of the spikelet of Avena eriantha. A: Upper glume (left), c. $23 \mathrm{~mm}$ long, and lower glume (right), B: Spikelet (c. $25 \mathrm{~mm}$ long), without glumes, C: Awned lemmas, D: Hairy callus.

En cuanto a su comportamiento ecológico (Ladizinsky, 1971; Baum, 1977; Danin \& FragmanSapir, 2016), los ejemplares fueron encontrados en una zona de pendiente muy tendida, de unión entre una paramera calcárea y la parte superior de una cuesta margosa con presencia de yeso. La población se hallaba en un claro, en el interior de una mancha mixta de encina [Quercus ilex subsp. ballota (Desf.) Samp.] y quejigo (Quercus faginea Lam. subsp. faginea), salpicada con algunos pinos de repoblación (Pinus halepensis Mill.). Algunas de las especies más representativas con las que compartía hábitat en el momento de la recolección (de las que recogimos muestras que están depositadas en el herbario personal del autor de este trabajo) eran: Achillea odorata L., Aegilops 
geniculata Roth, Aegilops triuncialis L., Convolvulus lineataus L., Dianthus pungens subsp. hispanicus (Asso) O. Bolòs \& Vigo, Helianthemum hirtum (L.) Mill., Lomelosia divaricata (Jacq.) Greuter \& Burdet, Salvia lavandulifolia Vahl subsp. lavandulifolia, Sideritis hirsuta L., Stipa juncea L., Thrincia hispida Roth y Xeranthemum inapertum (L.) Mill. Un problema importante de esta población (en lo que conocemos, de dimensiones bastante restringidas) es que, por hallarse aledaña a un camino, es una zona ideal para el descanso de vehículos, bien agrícolas, para los cultivos cerealistas de los páramos circundantes, bien particulares, para los cazadores que se acercan a la zona.

Teniendo en cuenta lo que se conoce sobre la distribución de la especie en la península, ya desde la aparición del Atlas y Libro Rojo de la Flora Vascular Amenzada de España (Bañares et al., 2004), la especie se ha incluido en la categoría DD, es decir, es una especie de la que se tienen datos insuficientes, no disponiéndose de un mínimo de información para evaluarla. De la misma manera ha sido considerada en las Listas Rojas de la Flora Vascular Española (Moreno, 2008, 2011). Desde un punto de vista nacional, a nivel legal (BOE, 2001) tampoco se ha hecho ninguna alusión a ella. En aquellas comunidades autónomas en donde Avena eriantha se conoce desde hace algunos años, el taxón tampoco ha sido incluido en los correspondientes catálogos de flora amenazada, ni se ha recogido a nivel legislativo: Andalucía (Blanca et. al., 1999, 2000; BOJA, 2012), Aragón (Alcántara de la Fuente, 2007; BOA, 1995, 2005), Castilla-La Mancha (DOCM, 1998, 2001) y Madrid (BOCM, 1992). Sólo de manera relativamente reciente (Cabezudo et al., 2005), en Andalucía, y también con la categoría de "Especie con Datos Insuficientes (DD)", ha sido incluida en una Lista Roja.

No podemos concluir este trabajo sin dejar de recordar, de nuevo, a Paunero (1958), que hace más de medio siglo ya insistía en la necesidad de hacer también recolecciones de plantas que pudieran parecernos a simple vista muy comunes y de escaso interés, ya que, en ocasiones, examinadas con detenimiento, resultan ser taxones no tan conocidos, como es el caso de la avena que nos ocupa.

\section{Agradecimientos}

A Samuel Pyke, quien amablemente nos proporcionó comentarios esclarecedores sobre la presencia de la especie en Aragón y el País Vasco.

\section{Bibliografía}

Alcántara de la Fuente, M. (Coord.) (2007). Catálogo de Especies Amenazadas en Aragón. Flora. Huesca: Gobierno de Aragón, Departamento de Medio Ambiente.
ANTHOS. (2019). Sistema de Información de las plantas de España. Real Jardín Botánico, CSICFundación Biodiversidad. Recurso electrónico en www.anthos.es [consultado el 02-XII-2019].

Bañares, A., Blanca, G., Güemes, J., Moreno, J.C. \& Ortiz, S. (Eds.) (2004). Atlas y Libro Rojo de la Flora Vascular Amenazada de España. Madrid: Dirección General de Conservación de la Naturaleza.

Baum, B.R. (1977). Oats: wild and cultivated. A monograph of the genus Avena L. (Poaceae). Otawa (Ontario, Canada): Byosistematic Research Institute, Canada Department of Agriculture, Research Branch.

Blanca, G., Cabezudo, B., Hernández-Bermejo, J.E., Herrera, C.M., Molero-Mesa, J., Muñoz, J. \& Valdés, B. (1999). Libro Rojo de la Flora Silvestre Amenazada de Andalucía. Tomo I: Especies en peligro de Extinción. Junta de Andalucía: Consejería de Medio Ambiente.

Blanca, G., Cabezudo, B., Hernández-Bermejo, J.E., Herrera, C.M., Muñoz, J. \& Valdés, B. (2000). Libro Rojo de la Flora Silvestre Amenazada de Andalucía. Tomo II: Especies Vulnerables. Junta de Andalucía: Consejería de Medio Ambiente.

BOA (1995). Decreto 49/1995, de 28 de marzo, de la Diputación General de Aragón, por el que se regula el Catálogo de Especies Amenazadas de Aragón. Boletín Oficial de Aragón no 42, 07-IV1995, pp. 1270-1275.

BOA (2005). Decreto 181/2005, de 6 de septiembre, del Gobierno de Aragón, por el que se modifica parcialmente el Decreto 49/1995, de 28 de marzo, de la Diputación General de Aragón, por el que se regula el Catálogo de Especies Amenazadas de Aragón. Boletín Oficial de Aragón no 114, 23-IX-2005, pp. 11527-11532.

BOCM (1992). Decreto 18/92, de 26 de marzo, por el que se aprueba el Catálogo Regional de especies amenazadas de fauna y flora silvestres y se crea la categoría de árboles singulares. Boletín Oficial de la Comunidad de Madrid no 85, 09-IV-1992, pp. 05-11.

BOE (2011). Real Decreto 139/2011, de 4 de febrero, para el desarrollo del Listado de Especies Silvestres en Régimen de Protección Especial y del Catálogo Español de Especies Amenazadas. Boletín Oficial del Estado ํo 46, 23-II-2011, pp. 20912-20951.

BOJA (2012). Decreto 23/2012, de 14 de febrero, por el que se regula la conservación y el uso sostenible de la flora y la fauna silvestres y sus hábitats. Boletín Oficial de la Junta de Andalucía no 60, 27-III-2012, pp. 114-163.

Cabezudo, B., Devesa, J.A., Tormo, R., Vázquez, F. \& Nieto-Caldera, J.M. (1990). Catálogo de las gramíneas malacitanas. Acta Botanica Malacitana, 15, 91-123.

Cabezudo, B., Talavera, S., Blanca, G., Salazar, C., Cueto, M., Valdés, B., Hernández-Bermejo, J.E., Herrera, C.M., Rodríguez-Hiraldo, C. \& Navas, D. (2005). Lista Roja de la flora vascular de Andalucía. Junta de Andalucía: Consejería de Medio Ambiente. 
Carrasco, M.A., Costa-Tenorio, M., JiménezAlbarrán, J., Prada-Moral, C., Velayos-Rodríguez, M. (1986). Catálogo de plantas vasculares. Trabajos del Departamento de Botánica, 13, 2337.

Carrasco, M.A., Macía, M.J. \& Velayos, M. (1997). Listado de plantas vasculares de Guadalajara. Valencia: Monografías de Flora Montiberica.

Cebolla-Lozano, C., López-Rodríguez, J.A., PostigoMijarra, J.M. \& Rivas-Ponce, M.A. (1997). Matritensis Plantarum Vascularium Catalogus. Poaceae. Fontqueria, 44, 1-86.

Cebolla-Lozano, C. \&Postigo-Mijarra, J.M. (1996). Avena eriantha Durieu, Mapa 734. Fontqueria, 44, 185-186.

Danin, A. \& Fragman-Sapir, O. (2016). Flora of Israel Online. Recurso electrónico en http://flora.org.il/en/plants/.

DOCM (1998). Decreto 33/1998, de 05-05-98, por el que se crea el Catálogo Regional de Especies Amenazadas de Castilla-La Mancha. Diario Oficial de Castilla-La Mancha № 22, 15-V-1998, pp. 3391-3398.

DOCM (2001). Decreto 200/2001, de 06-11-2001, por el que se modifica el Catálogo Regional de Especies Amenazadas. Diario Oficial de CastillaLa Mancha no 119, 13-XI-2001, pp. 12825-12827.

Dogan, M. (1985). Avena L. In P.H. Davis (Ed.), Flora of Turkey and the East Aegean Islands, Volume 9 (pp. 302-307). Edinburgh: Ediburgh University Press.

González-Bernáldez, F. (1997). Gramíneas pratenses de Madrid. Madrid: Comunidad de Madrid, Consejeríadel Medio Ambiente y Desarrollo Regional.

Hernández-Palacios, G. (2013). Notas sobre flora vascular de la provincia de Toledo (Península Ibérica, España). Anales de Biología, 35, 2940.doi: http://dx.doi.org/10.6018/analesbio.0.35.5

IPNI (2019). The International Plant Name Index, The Royal Botanic Gardens (Kew), Harvard University Herbaria and Australian National Herbarium. Recurso electrónico en http://www.ipni.org [consultado el 02-XII-2019].

Ladizinsky, G. (1971). Biological flora of Israel 2. Avena L. Israel Journal of Botany, 20, 133-151.

Montserrat-Recorder, P. \& Hernández-Cardona, A.M. (1983). Avena eriantha Durieu nueva para la flora ibérica. Anales del Jardín Botánico de Madrid, 39(2), 546.

Morales, R. (2003). Catálogo de plantas vasculares de la Comunidad de Madrid. Botanica Complutensis, 27, 31-70.

Moreno, J.C. (Coord.) (2008). Lista Roja 2008 de la flora vascular española. Madrid: Dirección General de Medio Natural y Política Forestal (Ministerio de Medio Ambiente, y Medio Rural y Marino, y Sociedad Española de la Conservación de Plantas).

Moreno, J.C. (Coord.) (2011). Lista Roja de la flora vascular española. Actualización con los datos de la Adenda 2010 al Atlas y Libro Rojo de la Flora Vascular Amenazada. Madrid: Dirección General de Medio Natural y Política Forestal (Ministerio de Medio Ambiente, y Medio Rural y Marino, y Sociedad Española de la Conservación de Plantas).

Paunero, E. (1958). Las Aveneas españolas, III. Anales del Jardín Botánico de Madrid, 15, 377415.

POWO (2019). Plants of the World online. The Royal Botanic Gardens (Kew). Recurso electrónico en http://www.plantsoftheworldonline.org/ [consultado el 02-XII-2019].

Prieto-Bocanegra, V. \& Fernández-López, C. (1992). Catálogo florístico de la provincia de Málaga con nomenclatura simplificada. Blancoana, 10, 47-55.

Pyke, S. (2005). Nota sobre la presencia de Avena eriantha Durieu en Aragón. Flora montiberica, 30, 41-42.

Rocha-Alfonso, M.L. (1980). Avena L. In T.G. Tutin, V.H. Heywood, N.A. Burges, D.M. Moore, D.H. Valentine, S.M. Walters \& D.A. Webb (Eds.), Flora Europaea, Volume 5, Alismataceae to Orchidaceae (Monocotyledones) (pp. 206-208). Cambridge: Cambridge University Press.

Romero-Zarco, C. (1983). Sobre la presencia en España de Avena eriantha Durieu. Anales de Jardín Botánico de Madrid, 40(1), 284.

Romero-Zarco, C. (1990). Claves para la identificación de los géneros de Gramíneas de la península Ibérica e Islas Baleares. Lagascalia, 15(2), 223-261.

Romero-Zarco, C. (1996). Sinopsis del género Avena L. (Poaceae, Aveneae) en España peninsular y Baleares. Lagascalia, 18, 171-198.

Romero-Zarco, C. (2011). Avena L. In G. Blanca, B. Cabezudo, M. Cueto, C. Morales-Torres \& C. Salazar (Eds.), Flora Vascular de Andalucía Oriental (pp. 321-323). Universidades de Almería, Granada, Jaén y Málaga (España).

Romero-Zarco, C. (2015). Las Gramíneas de la península Ibérica e Islas Baleares. Claves ilustradas para la determinación de los géneros y catálogo preliminar de las especies. Jaca: Jolube Consultor Botánico y Editor.

Sánchez-Gómez, P., López-García, D., Cánovas, J.L., Jiménez, J.L., Vera, J.B., \& Martínez-López, P. (2018). Novedades florísticas para las provincias de Murcia, Albacete y Almería (España). Anales de Biología, 40, 73-85. doi: http://dx.doi.org/10.6018/analesbio.40.09

Sanz-Elorza, M. (2006). Aproximación al catálogo florístico de la provincia de Toledo. Ecología, 20, 89-116.

Tutin, T.G., Heywood, V.H., Burges, N.A., Moore, D.M., Valentine, D.H., Walters, S.M. \&Webb, D.A. (Eds.), Flora Europaea, Volume 5, Alismataceae to Orchidaceae (Monocotyledones). Cambridge: Cambridge University Press. 